05

\title{
Энергетические параметры активационных процессов в области фазовых превращений в нанокластере суперионного проводника $\mathrm{LaF}_{3}$
}

\author{
() В.Ф. Криворотов, Г.С. Нуждов
}

Институт ионно-плазменных и лазерных технологий АН Узбекистана, 100125 Ташкент, Узбекистан

e-mail: valeriy.1980@mail.ru

(Поступило в Редакцию 6 апреля 2016 г.)

Представлены результаты квантово-химических расчетов профилей внутриячеечного потенциального рельефа при элементарном акте разупорядочения в различных частях решетки кристаллита $\mathrm{LaF}_{3}$ размером $3.5 \times 2.0 \times 2.2 \mathrm{~nm}$, содержащего 1200 ионов. Для диэлектрической и высокопроводящей фаз установлено, что потенциальные барьеры, ограничивающие движение в анионной подрешетке, в центральной части нанокластера примерно в 1.5-2.0 раза превышают потенциальные барьеры на его гранях. Показано, что по этой причине эффективность термостимулированного движения в анионной подрешетке на гранях кристаллита на несколько порядков превосходит эффективность движения ионов фтора в его центральных областях.

DOI: 10.21883/JTF.2017.04.44311.1839

\section{Введение}

Частным случаем в широком классе ионопроводящих соединений являются суперионные (СИ) кристаллы особый класс твердых материалов, в которых фазовые превращения (ФП) связаны с температурным разупорядочением одной из кристаллических подрешеток и определяются переходом системы ионов из диэлектрического (ДЭ) состояния в СИ. В первом приближении в СИ фазе они представляют собой „жидкость“ подвижных ионов, текушую в подрешетке неподвижных ионов. С точки зрения фундаментальных процессов, в частности процессов переноса, эти материалы занимают промежуточное положение между жидкостью и твердым телом.

В качестве объекта исследования выбран трифторид лантана $\mathrm{LaF}_{3}$ и его аналоги структурного типа тисонита $-\mathrm{LnF}_{3}(\mathrm{Ln}=\mathrm{La}, \mathrm{Ce}, \mathrm{Pr})$, представляющие собой типичные твердые электролиты с проводимостью порядка $10^{-5}-10^{-3} \mathrm{~S} / \mathrm{cm}[1-3]$, которая примерно на 5-7 порядков превосходит проводимости обычных ионных кристаллов. В кристаллах $\mathrm{LnF}_{3}$ переход из ДЭ фазы в СИ размыт в некотором интервале температур и не сопровождается значительной перестройкой структуры кристаллической решетки [4,5]. Например, разупорядочение анионной подрешетки в кристалле $\mathrm{LaF}_{3}$ происходит в области температур $\approx 180-460 \mathrm{~K}$ и продолжается до температуры плавления решетки. При этом в области $\approx 180-300 \mathrm{~K}$ разупорядочение решетки определяется движением в подрешетке ионов $\mathrm{F}_{1}$, составляющих $2 / 3$ от общего числа ионов фтора. Оставшиеся ионы фтоpa $F_{2}$ и $F_{3}$ образуют вместе с ионами металла базовые анионно-катионные плоскости. Подрешетки этих ионов разупорядочиваются при температурах, превышающих $400-420 \mathrm{~K}[1,2]$.
Интерес, проявляемый специалистами по исследованию процессов переноса, к $\mathrm{LnF}_{3}$, в первую очередь, определяется широкими возможностями применения этих кристаллов в различных технических областях: в качестве материала для рабочих модулей в твердотельных батареях, электрохромных дисплеях, электрохимических сенсорах, газоаналитических датчиках и различных оптоионных устройствах [6-8]. Достаточно важным фактором в различных технических приложениях выступает и то обстоятельство, что сравнительно высокой ионной проводимостью кристаллы $\mathrm{LnF}_{3}$ обладают уже при комнатной температуре $(T \approx 300 \mathrm{~K})$.

К основным параметрам переноса ионов относятся энергии $E_{a}$ образования точечных дефектов типа вакансия - межузельный ион и величины потенциальных барьеров $E_{m}$, ограничивающих ячеечные перемещения ионов фтора. К настоящему времени известно $[1,9,10]$, что, к примеру, в кристалле $\mathrm{LaF}_{3}$ характерные для $E_{a}$ и $E_{m}$ значения энергии активации лежат в интервале $0.20-0.60 \mathrm{eV}$. Понятно, что такие различающиеся значения энергий $E_{a}$ и $E_{m}$ требуют новых модельных подходов к механизмам „плавления“ анионной подрешетки и иной интерпретации полученных результатов, а также дополнительных экспериментальных исследований с применением более „тонких“ методик и приемов.

В настоящей работе при помощи пакета квантовохимических программ МОРАС 2012 [11] рассчитаны величины барьеров и минимумов на пути движения ионов $\mathrm{F}_{1}$, перемещающихся в ближайшее междоузлие в различных частях кристаллита $\mathrm{LaF}_{3}$, a именно в центральной части кластера, а также в области его поверхности. При этом квантово-химические расчеты энергетических параметров $E_{a}$ и $E_{m}$ в случае поверхностных перемещений ионов фтора выполнены впервые 


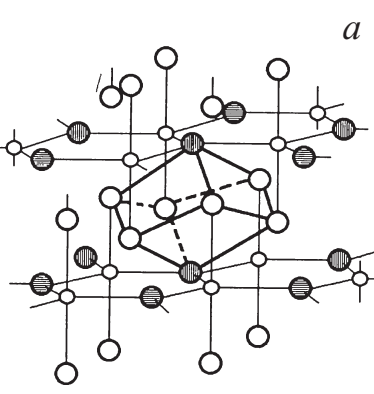

$a$

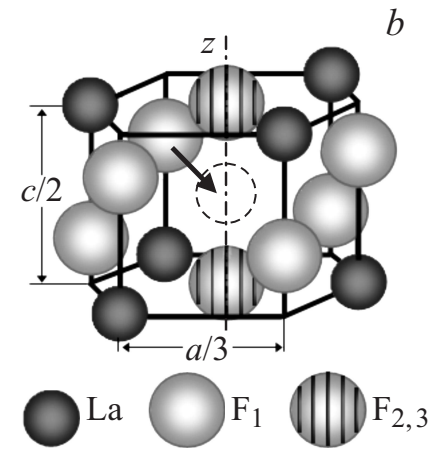

Рис. 1. Фрагмент решетки $\mathrm{LaF}_{3}$, включающий ромбоэдрическое междоузлие между анионно-катионными плоскостями $(a)$ и иллюстрация перемещения в него одного из ионов $\mathrm{F}_{1}(b)$.

для двух фазовых состояний $\mathrm{LaF}_{3}$ - для ДЭ и СИ фаз. Методика расчета профилей внутриячеечных потенциальных рельефов в нанокластере $\mathrm{LaF}_{3}$ при перемещении ионов фтора из узельной позиции в ближайшее междоузлие достаточно подробно описана в [12]. В этой же работе показано, что уже при числе частиц в кластеpe $\mathrm{LaF}_{3}$ порядка 600-800 активационные энергии $\mathrm{E}_{a}$ и $E_{m}$ принимают значения, практически совпадающие со значениями для объемных образцов $\mathrm{LaF}_{3}$. По этой причине для проведения расчетов и получения корректных результатов был выбран кластер „кубической“ формы размером $3.5 \times 2.0 \times 2.2 \mathrm{~nm}$, содержащий 1200 атомов.

В $[10,13]$ показано, что разупорядочение анионной подрешетки в кристаллах $\mathrm{LnF}_{3}$ происходит путем теплового перемещения ионов фтора в энергетически эквивалентные междоузлия, расположенные между анионнокатионными плоскостями, с образованием массива точечных дефектов типа анионная вакансия-межузельный анион. При этом максимально разупорядочивается лишь около $17 \%$ анионной подрешетки, хотя понятно, что во внутреннем движении участвуют все ионы фтора [13].

На рис. $1, a$ приведен фрагмент решетки $\mathrm{LaF}_{3}$, содержащий такое ромбоэдрическое междоузлие. Хорошо видно, что в плоскости $X Y$ междоузлие ограничено двумя тройками ионов $F_{1}$, образующими шестичленное гофрированное кольцо. В направлении оси $Z$ междоузлие ограничено ионами $\mathrm{F}_{2}$ или $\mathrm{F}_{3}$. Рис. $1, b$ иллюстрирует перемещение одного из шести ионов $\mathrm{F}_{1}$ в такое междоузлие. Очевидно, что если междоузлие расположено в центре нанокластера, то все шесть ионов $\mathrm{F}_{1}$, формирующих его в плоскости $X Y$, эквивалентны относительно перемещения в междоузлие.

\section{Результаты и обсуждение}

Профили потенциальных рельефов были рассчитаны для следующих типов перемещения ионов $\mathrm{F}_{1}$. В первом случае ион $\mathrm{F}_{1}$ был выбран в центре кластера и его перемещение в междоузлие происходило параллельно постоянной решетки $a$ (параллельно кристаллографической оси $X$ ). В другом случае перемещение иона в междоузлие осуществлялось в плоскости грани $X Y$, параллельной анионно-катионным плоскостям. В третьем случае перемещение иона $F_{1}$ в междоузлие происходило также параллельно постоянной решетки $a$, но в плоскости грани $X Z$, перпендикулярной анионно-катионным плоскостям.

На рис. 2 схематично показаны направления различных типов перемещения ионов $F_{1}$ в решетке нанокластера $\mathrm{LaF}_{3}$. Стрелки показывают направление прыжка ионов $F_{1}$ из узельных позиций решетки в междоузлия в центральной части кластера $(1)$ и на различных его гранях (2 и 3). Цифры $1-3$ соответствуют кривым $1-3$ на рис. 3 , который иллюстрирует результаты расчетов профилей потенциальных рельефов в ДЭ фазе кристалла $\mathrm{LaF}_{3}$, связанных с перемещением отдельных (изолированных) ионов $\mathrm{F}_{1}$ в различных частях кластера. Кривая 1, приведенная для сравнения, описывает профиль потенциального рельефа в ДЭ фазе $\mathrm{LaF}_{3}$ при перемещении иона $F_{1}$ в междоузлие в центральной части

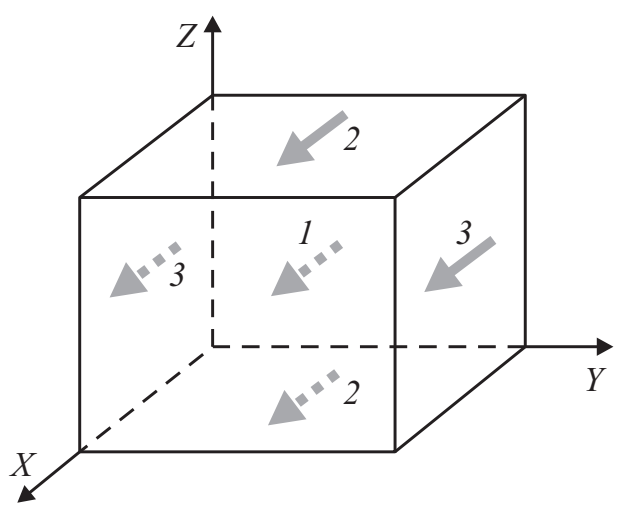

Рис. 2. Схематичное представление различных перемещений ионов $\mathrm{F}_{1}$ в решетке нанокластера $\mathrm{LaF}_{3}$.

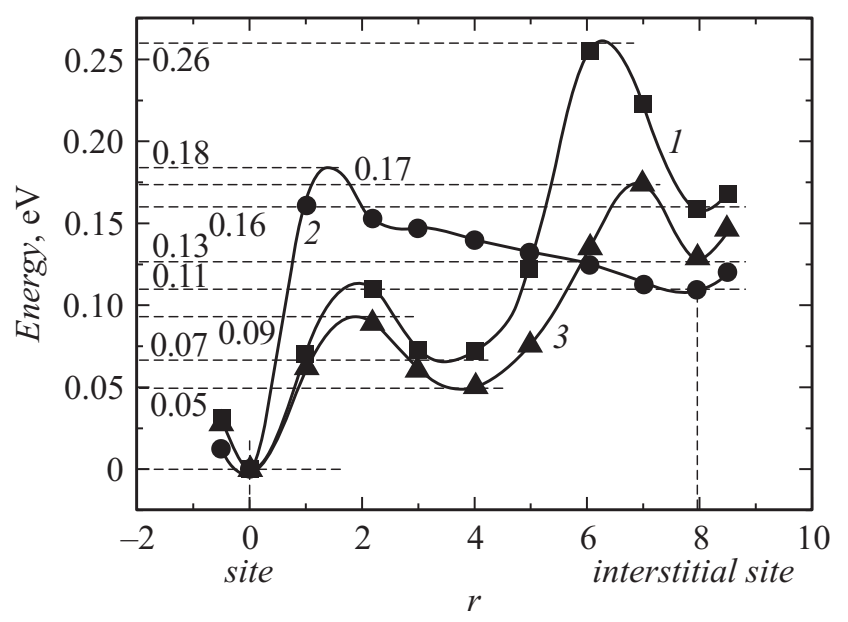

Рис. 3. Профили потенциальных рельефов в ДЭ фазе при перемещении иона $\mathrm{F}_{1}$ из узельной позиции в междоузлие в различных областях нанокластера $\mathrm{LaF}_{3}$. 
кластера; кривые 2 и 3 показывают профили потенциальных рельефов, связанные с перемещением в междоузлия отдельных поверхностных ионов $\mathrm{F}_{1}$, расположенных на двух смежных гранях кластера. При проведении расчетов перемещающиеся ионы $\mathrm{F}_{1}$ выбирались по возможности в центральных частях граней. Понятно, что противоположные грани „кубической“ решетки структурно эквивалентны, и значит потенциальные профили на них описываются одинаковыми кривыми.

Анализ кривых 2 и 3 и соответствующих барьеров $E_{m}$ позволяет заключить, что для ДЭ фазы поверхностные прыжки ионов $\mathrm{F}_{1}$ вдоль грани $X Y$ в соответствии с соотношением $v_{i} \sim v_{0} \exp \left(-E_{m} / k T\right)$ реализуются значительно активнее (при прочих равных условиях), чем в центральных областях нанорешетки. Здесь $v_{0}-$ узельная частота колебаний иона $\mathrm{F}_{1}, v_{i}$ - частота его попадания на вершину барьера $E_{m}$. Величина эффективного барьера в центральной части решетки есть $E_{m}=0.37 \mathrm{eV}$ $(0.11 \mathrm{eV}+0.26 \mathrm{eV})$ (рис. 3, кривая 1$)$, тогда как величина барьера на грани $X Y$ (кривая 2) есть $E_{m}=0.18 \mathrm{eV}$. Видно, что величины потенциальных барьеров различаются более чем в 2 раза, что обеспечивает увеличение эффективности прыжков ионов $\mathrm{F}_{1}$ на грани $X Y$ более чем на 3 порядка. В связи с вышеприведенным значением барьера $E_{m}=0.37 \mathrm{eV}$ заметим, что однозначно не установлено точного критерия определения эффективной величины нескольких близко расположенных барьеров на пути перемещения мобильной частицы [14]. Относительно разумным представляется положение, по которому за эффективное значение нескольких близких барьеров в первом приближении можно принять их суммарную величину, что и было сделано выше.

Совершенно иной вид имеет потенциальный профиль в ДЭ фазе при перемещении иона $\mathrm{F}_{1}$ вдоль грани $X Z$ нанорешетки, перпендикулярной анионно-катионным плоскостям (кривая 3 на рис. 3). В этом случае эффективный барьер $E_{m}=0.26 \mathrm{eV} \quad(0.09 \mathrm{eV}+0.17 \mathrm{eV})$. Несложный расчет с учетом приведенного выше соотношения для частоты $v_{i}$ показывает, что прыжки ионов $\mathrm{F}_{1}$ по грани $X Z$ для ДЭ состояния кристалла $\mathrm{LaF}_{3}$ осуществляются примерно на 2 порядка чаще, чем в центральных областях нанорешетки. Таким образом, наиболее активный (массовый) перенос ионов $\mathrm{F}_{1}$ в направлении кристаллографической оси $X$ для ДЭ состояния наблюдается на боковых (относительно направления движения ионов $\mathrm{F}_{1}$ ) гранях кристалла $\mathrm{LaF}_{3}$. Следовательно, ВП состояние, в первую очередь, наступает в поверхностных областях нанокластера $\mathrm{LaF}_{3}$, с ростом температуры распространяясь на весь его объем.

На рис. 4 приводятся результаты расчетов профилей потенциального рельефа, связанных с перемещением отдельных ионов $\mathrm{F}_{1}$ в различных частях нанокластера $\mathrm{LaF}_{3}$, находящегося в СИ состоянии. Кривая 1 описывает профиль потенциального рельефа в СИ фазе $\mathrm{LaF}_{3}$ при перемещении иона $\mathrm{F}_{1}$ в междоузлие в центральной части кластера. Кривые 2 и 3 описывают профили

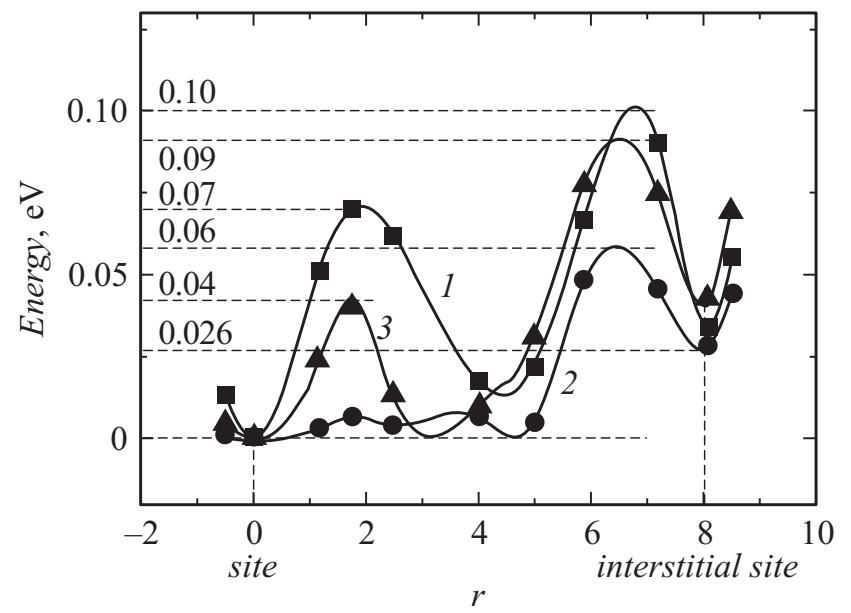

Рис. 4. Профили потенциальных рельефов в СИ фазе при перемещении иона $\mathrm{F}_{1}$ из узельной позиции в междоузлие в различных областях нанокластера $\mathrm{LaF}_{3}$.

потенциальных рельефов в СИ фазе, связанные с перемещением в междоузлия поверхностных ионов $\mathrm{F}_{1}$. Хорошо видно, что для случаев перемещения ионов $\mathrm{F}_{1}$ по граням кристаллита величины эффективных барьеров $E_{m}$ существенно меньше значения эффективного барьера, ограничивающего движение иона $\mathrm{F}_{1}$ в центре решетки. В случае движения по грани $X Y$ (кривая 2) в СИ фазе величина барьера $E_{m}$ равна $0.06 \mathrm{eV}$, что в 3 раза меньше, чем величина барьера $E_{m}=0.18 \mathrm{eV}$ в ДЭ состоянии кристалла $\mathrm{LaF}_{3}$ (рис. 3). Такое значительное уменьшение барьера при переходе в ВП состояние хорошо согласуется с известным в физике СИ проводников положением, по которому в ВП фазе внутриячеечное движение ионов происходит значительно эффективнее, чем в ДЭ состоянии кристалла. При движении иона $\mathrm{F}_{1}$ по смежной грани $X Z$ (кривая 3 на рис. 4) барьер $E_{m}$ составляет $0.13 \mathrm{eV}(0.04 \mathrm{eV}+0.09 \mathrm{eV})$, тогда как в ДЭ фазе этот барьер составлял $0.26 \mathrm{eV}$. Простые оценки частоты $v_{i}$ реализованных прыжков показывают, что при переходе в СИ фазу барьер, ограничивающий перемещение иона $\mathrm{F}_{1}$ вдоль грани $X Z$, уменьшается в 2 раза. Это обстоятельство приводит к увеличению эффективности ионного переноса на грани $X Z$ почти на 3 порядка. Квантово-химические расчеты показали, что в СИ фазе величина барьера $E_{m}$ в центральной части кластера составляет $0.17 \mathrm{eV}(0.07 \mathrm{eV}+0.10 \mathrm{eV})$, тогда как в ДЭ фазе барьер составлял $E_{m}=0.37 \mathrm{eV}(0.11 \mathrm{eV}+0.26 \mathrm{eV})$. Следовательно, барьер $E_{m}$ в центре нанорешетки при переходе в СИ состояние уменьшается более чем в 2 раза, что соответствует увеличению эффективности движения в подрешетке ионов $\mathrm{F}_{1}$ более чем на 3 порядка.

Таким образом, полученные данные позволяют заключить, что в СИ фазе $\mathrm{LaF}_{3}$ частота прыжков ионов фтора $\mathrm{F}_{1}$ на его гранях на несколько порядков превосходит частоту прыжков в его центральной части. Разупорядочение подрешеток $\mathrm{F}_{2}$ и $\mathrm{F}_{3}$ в работе не рассматривается, 
поскольку соответствующие им ионы фтора составляют лишь около $30 \%$ анионной подрешетки.

Похожим образом ведут себя активационные энергии $E_{a}$, определяющие приращение решеточной энергии кристалла при перемещении иона из узла, например, в междоузлие (восьмая позиция на рис. 3 и 4). Хорошо видно, что для СИ состояния кристалла $\mathrm{LaF}_{3}$ значения $E_{a}$ лежат в интервале $\approx 0.03-0.04 \mathrm{eV}$ (рис. 4) при перемещении ионов $\mathrm{F}_{1}$ в различных частях кластера. Для ДЭ же состояния кристалла $\mathrm{LaF}_{3}$ (рис. 3) значения $E_{a}$ при перемещении иона фтора в центральных областях нанокластера в среднем на $25 \%$ превышают значения $E_{a}$ для его поверхностных областей, из чего следует, что с ростом температуры процесс разупорядочения решетки $\mathrm{LaF}_{3}$ вначале активизируется на поверхности нанокластера. Такой же вывод был сделан выше при рассмотрении потенциальных барьеров $E_{m}$. Для случая перемещения ионов $\mathrm{F}_{1}$ в ДЭ фазе кристаллита $\mathrm{LaF}_{3}$ значениям $E_{a}$ соответствуют, как и следовало ожидать [6], значительно бо́льшие энергии в интервале $0.11-0.16 \mathrm{eV}$ (рис. 3). Проведенные квантово-химические расчеты позволяют заключить, что как в ДЭ, так и в СИ фазе движение ионов $\mathrm{F}_{1}$ по поверхности нанокластера происходит на несколько порядков эффективнее, чем перемещение ионов фтора в его центральных частях. Между тем этот вывод можно назвать достаточно ожидаемым, поскольку число межчастичных связей для поверхностных ионов, как правило, заметно меньше числа связей для ионов, расположенных во внутренних областях решетки, из чего следует, что ионам, расположенным на поверхности кристаллита, соответствуют значительно меньшие значения энергии связи. Таким образом, впервые квантовохимическими расчетами для ВП состояния $\mathrm{LaF}_{3}$ установлен эффект аномально высокой подвижности ионов фтора на поверхности нанокластера по сравнению с движением ионов фтора в его центральных частях.

Все сказанное выше позволяет заключить, что, возможно, уже в недалекой перспективе при разработке конструкционных материалов, например для батарей и аккумуляторов с высокими плотностями тока вместо ионопроводящих элементов цилиндрической и прямоугольной формы, будут использовать трубчатые наноконструкции (практически без потери плотности электрического тока). Иными словами, описанный эффект аномально высокой подвижности ионов фтора на поверхности нанокластера может оказаться определяющим фактором при разработке рабочих элементов на основе наноструктурированных ионопроводящих материалов в различных технических устройствах микроэлектроники.

\section{Заключение}

В настоящей работе сделана попытка показать изменение энергетических параметров, определяющих эффективность переноса ионов фтора, при их перемещении в различных областях нанорешетки СИ кристалла $\mathrm{LaF}_{3}$, a также показать зависимость этих параметров от степени ее разупорядочения, в частности, при переходе из ДЭ состояния в СИ фазу.

Анализ работ последних лет показал, что впрямую описать зависимость энергетических параметров, характеризующих эффективность внутриячеечного переноса ионов фтора от степени разупорядочения решетки кристалла $\mathrm{LaF}_{3}$, к примеру, в рамках классической термодинамики, достаточно сложно [6]. Как правило, используемые при этом физические подходы и математические приемы приводят, с одной стороны, к сложным математическим выкладкам, связанным с флуктуационным характером процесса образования точечных дефектов типа узельная вакансия-межузельный ион, а с другой к существенному увеличению компьютерного времени моделирования. Между тем с развитием компьютерной техники и современного программного обеспечения проблема описания связи величины энергетических параметров $E_{a}$ и $E_{m}$ с различными типами разупорядочения нанорешетки $\mathrm{LaF}_{3}$ может быть в значительной мере разрешена. Под различными типами разупорядочения нанорешетки следует понимать перемещение ионов фтора в различных ее областях (в нашем случае - в центральной части нанорешетки и вдоль ее поверхностей). Особо отметим то обстоятельство, что современное компьютерное моделирование дает уникальную возможность на микроскопическом (атомарном) уровне исследовать особенности внутриячеечного движения отдельных ионов в широком интервале температур (конечно, при условии, что заданная кристаллическая структура реально отражает особенности моделируемой решетки твердого тела).

Более того, квантово-химическое моделирование процессов перемещения ионов в разупорядочивающейся подрешетке в ряде случаев может обеспечить выявление новой более детальной информации о диффузионном движении ионов, получение которой в лабораторных экспериментах очень затруднено либо просто невозможно. По этой причине получаемые посредством квантовохимического моделирования данные, как правило, являются оригинальными, показывающими атомарную картину, например активационных процессов в решетке СИ проводника.

Анализ описанной выше картины процесса разупорядочения в различных частях нанорешетки $\mathrm{LaF}_{3}$ позволяет сделать следующие заключения и обобщения.

1. Двухкомпонентные СИ кристаллы $\mathrm{LnF}_{3}$ структурного типа тисонита показали себя удобными и привлекательными модельными системами для детального исследования энергетических характеристик внутреннего движения в ионопроводящих материалах. Проведенные расчеты позволили на микроскопическом уровне получить новые результаты по описанию высокой анионной подвижности в нанорешетке $\mathrm{LaF}_{3}$.

2. Разработанные авторами для нанорешетки кристалла $\mathrm{LaF}_{3}$ модельные приемы получения микроскопически малых значений (порядка сотых долей 
электрон-вольт $\mathrm{eV}$ ) энергетических параметров $E_{a}$ и $E_{m}$, детально описывающих профиль внутриячеечного перемещения ионов $\mathrm{F}_{1}$, применимы к описанию энергетических характеристик диффузионного движения ионов в обширном классе СИ проводников с размытыми фазовыми переходами. Следует особо отметить, что описанные в настоящей работе энергетические параметры $E_{a}$ и $E_{m}$ достаточно хорошо согласуются со значениями этих параметров, полученных другими методами, например, из экспериментов по неупругому рассеянию света $[13,15]$.

3. К одному из основных выводов, полученных с помощью расчетов по квантово-химическим программам МОРАС 2012, следует отнести заключение о том что, в СИ фазе потенциальные барьеры в „квазижидкой“ подрешетке в некоторых кристаллографических направлениях действительно уменьшаются в несколько раз (относительно потенциального рельефа в бездефектной решетке). Таким образом, основная часть результатов, впервые полученных для кристалла $\mathrm{LaF}_{3}$, представляет несомненный интерес для физики СИ состояния и в более широком плане - для физики конденсированных сред в целом.

Работа выполнена при финансовой поддержке Государственной программы фундаментальных исследований Академии наук Республики Узбекистан (грант ФА-Ф2-Ф081) и Узбекско-Российского гранта MP-30-2008.

\section{Список литературы}

[1] Rhandour A., Reau J.M., Matar S.F. et al. // Mat. Res. Bull. 1985. Vol. 20. P. 1309-1327.

[2] Привалов А.Ф., Мурин И.В. // ФТТ. 1999. Т. 41. № 9. C. $1616-1620$.

[3] Mohamed El Omari, Jean Sénegas, Jean Maurice Réau // Solid State Ionics. 1998. Vol. 107. P. 281-291.

[4] Privalov A.F., Lips O., Fujara F. // J. Phys.: Condens. Matter. 2002. Vol. 14. P. 4515-4519.

[5] Гарашина Л.С., Соболев Б.П., Александров В.Б. и др. // Кристаллография. 1980. Т. 25. № 2. С. 294-299.

[6] Гуревич Ю.Я., Харкаи Ю.И. Суперионные проводники. М.: Наука. 1992. 244 с.

[7] Tarahashi T., Kuwabara K. Physics and Chemistry of Solid Electrolytes - Ed. I. Yokota — Niigata (Japan): Niigata University. 1990. P. 40-93.

[8] Потанин А.A. // Российский хим. журн. 2001. T. XLV. № 5-6. C. 58-63.

[9] Воронов Б.М., Волков С.В. // Электрохимия. 2004. Т. 40. № 1. C. 54-60.

[10] Krivorotov V.F., Khabibullaev P.K., Sharipov Kh.T. // Inorganic Materials. 2010. Vol. 46. № 6. P. 668-672 (in Russian).

[11] MOPAC 2012, James J.P. Stewart, Stewart Computational Chemistry, Colorado Springs, CO, USA, http://openmopac.net.

[12] Криворотов В.Ф., Нуждов Г.С. // ЖТФ. 2012. Т. 82. № 12. C. $58-62$.
[13] Криворотов В.Ф., Хабибуллаев П.К., Фридман А.А. и др. // Неорганические материалы. 2010. Т. 46. № 7. C. 875-879.

[14] John Manning. Diffusion Kinetics for Atoms in Crystals. Van Nostrand, Princeton, New Jersey, 1968, 256 p.

[15] Криворотов В.Ф., Фридман А.А., Чарная Е.В. Квазиупругое рассеяние света в суперионных кристаллах $\mathrm{LnF}_{3}$ $(\mathrm{Ln}=\mathrm{La}, \mathrm{Ce}, \mathrm{Pr})$ // Узбек. физич. журн. 2011. Т. 13. № 2. C. 104-109. 